Revista Brasileira de Agricultura Irrigada v.8, nº 1, p. 12 - 25 , 2014

ISSN 1982-7679 (On-line)

Fortaleza, CE, INOVAGRI - http://www.inovagri.org.br

DOI: $10.7127 /$ rbai.v8n100193

Protocolo 193.13 - 24/10/2013 Aprovado em 30/01/2014

\title{
AVALIAÇÃO TÉCNICA DE DOIS TIPOS DE EMISSORES EMPREGADOS NA IRRIGAÇÃO LOCALIZADA
}

\author{
Giuliani do Prado ${ }^{1}$, Lílian Henrique Nunes ${ }^{2}$, Adriano Catossi Tinos ${ }^{3}$
}

\begin{abstract}
RESUMO
O objetivo do trabalho foi avaliar através de ensaios de laboratório e campo, na Universidade Estadual de Maringá, em Cidade Gaúcha, PR, o desempenho de tubos gotejadores autocompensantes e não autocompensantes com emissores espaçados em um metro na linha. No laboratório de Hidráulica foi montada uma bancada de testes para estabelecer, no intervalo de pressão entre 5 a 30 mca, a equação característica e o coeficiente de variação de fabricação (CVF) dos emissores. No campo foram instaladas seis linhas laterais de tubos gotejadores com 61 metros de comprimento, que foram submetidos as pressões de operação de 5,10 e 15 mca, para a determinação das características de distribuição de água do sistema de irrigação. Expoentes de 0,1116 e 0,5403 da equação característica do emissor foram determinados, respectivamente, para tubos gotejadores autocompensantes e não autocompensantes. Em ambos os tubos gotejadores estudados foram observados CVF inferiores a 5\%, que classificam os emissores como excelentes. Na operação do sistema de irrigação no campo, uniformidades de distribuição de água (UD) entre $84,9 \%$ e $93,1 \%$ foram observadas para tubos gotejadores autocompensantes e entre $90,8 \%$ e $96,0 \%$ para tubos gotejadores não autocompensantes. Com exceção da UD $=84,9 \%$ dos tubos gotejadores autocompensantes sob a pressão $5 \mathrm{mca}$, os valores de UD encontraram-se dentro das recomendações sugeridas na literatura (UD > 85\%). Acréscimos na pressão de operação de 10 para 15 mca não proporcionaram melhoras na UD nem nos valores de eficiência de irrigação.
\end{abstract}

Palavras - chaves: tubo gotejador, pressão de operação, uniformidade de distribuição

\section{TECHNICAL EVALUATION OF TWO EMITTER TYPES USED IN DRIP IRRIGATION}

\footnotetext{
ABSTRACT

The work purpose was evaluate from laboratory and field tests, at Universidade Estadual de Maringá, in Cidade Gaúcha, PR, the performance of auto-compensated dripline and non-

${ }^{1}$ Engo Agrônomo, Doutor, Departamento de Engenharia Agrícola, Universidade Estadual de Maringá, Cidade Gaúcha - PR, CEP: 87.820-000, Fone: (0XX44) 3675 -1879, e-mail: gprado@uem.br

2 Eng ${ }^{\circ}$ Agrícola, IRRIGER, unidade de Unaí - MG, e-mail: lilian.h.nunes@hotmail.com

${ }^{3}$ Eng $^{\circ}$ Agrícola, Departamento de Engenharia Agrícola, Universidade Estadual de Maringá, e-mail: actinos@uem.br
} 
compensated dripline with inline emitters spaced at one meter. For establish the characteristic equation and manufacturer's coefficient of variation (CVM) of the emitters, from pressure interval of 5 to $30 \mathrm{mca}$, was assembled a test bench at the hydraulics laboratory. Six dripline laterals with 61 meter in length, operating under three different working pressures $(5,10$ and $15 \mathrm{mca})$, were installed at field for determining the water distribution characteristics of drip irrigation system. The characteristic equation showed exponents of 0.1116 and 0.5403 respectively, determined for auto-compensated emitters and non-compensated emitters. In both driplines studied were observed CVM lesser than 5\%, which classified the emitters as excellent. Water distribution uniformities (UD) between $84.9 \%$ and $93.1 \%$ were observed for auto-compensated emitters and between $90.8 \%$ and $96.0 \%$ for non-compensated emitters which operated in a drip irrigation system. UD values were within the recommendations suggested in the literature (UD > 85\%), the exception was the auto-compensated driplines operating under working pressure of $5 \mathrm{mca}(\mathrm{UD}=84.9 \%)$. Increasing working pressure from 10 to 15 mca did not provide improvements in UD or the efficiency irrigation values.

Keywords: dripline, working pressure, distribution uniformity

\section{INTRODUÇÃO}

Com a necessidade do emprego racional da água, a irrigação localizada vem conquistando cada vez mais espaço, devido a economia de água e energia que este método de irrigação proporciona. No levantamento dos equipamentos pressurizados, comercializados no período de 2001 a 2007, realizado pela Associação Brasileira de Máquinas e Equipamentos, houve um aumento da área irrigada com sistemas localizados de irrigação (PIRES et al., 2008). Conforme Saraiva e Souza (2012), esse método de irrigação vem sendo empregado de forma mais expressiva nas regiões Sudeste e Nordeste do país.

O menor consumo de água e energia, obtidos com os sistemas de irrigação localizada, está associado a aplicação pontual de água através de emissores (gotejadores ou microaspersores) que operam com baixas pressões de serviço. Desta maneira, os gotejadores apresentam-se como peças fundamentais dentro do sistema de irrigação por gotejamento (BERNARDO et al., 2006; MANTOVANI et al., 2009; BORSSOI et al., 2012).

Em função do expoente da equação potencial do gotejador, estes podem ser classificados como gotejadores de regime laminar, turbulento ou autocompensante (CABELLO, 1996), com um maior emprego no mercado nacional dos dois últimos tipos de emissores. Nos gotejadores autocompensantes, o expoente da equação característica tende a zero e para os gotejadores de regime turbulento este expoente tende a 0,5.

$\mathrm{O}$ valor do expoente da vazão do emissor influencia diretamente o comprimento das linhas laterais de irrigação. Nos gotejadores de regime autocompensante, a menor sensibilidade as variações da vazão, devido a variações de pressões, permite o dimensionamento de linhas laterais mais extensas, em relação a gotejadores que apresentam regime turbulento. Keller e Bliesner (1990) e Laperuta Neto et al. (2011) 
comentam que uma variação de vazão de $10 \%$, entre o primeiro e último emissor, é empregada no dimensionamento de linhas laterais de gotejadores. Entretanto, este critério pode ser alterado em função do retorno econômico da cultura.

Holzapfel et al. (2007), ao avaliar técnica e economicamente sistemas de irrigação localizada com gotejadores de regime turbulento e autocompensantes, verificaram que independentemente do tipo de emissor, sistemas operando com baixas pressões de serviço proporcionam menores custos totais anuais. Os autores observaram que emissores de regime turbulento apresentaram menor custo total anual, em relação aos autocompensantes, devido ao menor custo de aquisição e menor faixa de pressão utilizada na operação dos emissores.

O controle de qualidade na fabricação dos emissores é essencial, pois emissores que apresentam altos valores de coeficiente de variação de fabricação proporcionam grandes diferenças de vazões entre emissores (SOUZA et al., 2012). Segundo as normas da American Society of Agricultural Engenieers (2003), os emissores são classificados de acordo com o coeficiente de variação de fabricação (CVF) como: excelente (CVF $<5 \%$ ); médio ( $\leq$ CVF $<7 \%$ ); marginal $(7 \leq \mathrm{CVF}<11 \%)$; ruim $(11 \leq \mathrm{CVF}<15 \%)$ e; inaceitável ( $C V F \leq 15 \%)$. Como a equação para estimativa da uniformidade de emissão (ASAE, 2003) é função do CVF, emissores com baixos valores de CVF geram maiores valores de uniformidade de emissão.

Sistemas de irrigação localizada, dimensionados adequadamente e operando com emissores de alta qualidade, quando instalados no campo, podem sofrer a influência de fatores (temperatura, topografia e entupimentos) não considerados na concepção do projeto. Para avaliar o efeito desses fatores na qualidade da distribuição de água do sistema de irrigação, ensaios de campo devem ser executados. Conforme Soccol et al. (2002), parâmetros de desempenho, expressos pelo coeficiente de uniformidade de distribuição de água, eficiência de irrigação e área adequadamente irrigada, refletem a qualidade da irrigação.

Para o bom desempenho e o manejo de um sistema de irrigação localizada, é imprescindível o conhecimento das características hidráulicas dos emissores e seu comportamento no campo. Desta forma, o objetivo do trabalho foi avaliar, em laboratório e em campo, o desempenho de tubos gotejadores de regime turbulento e autocompensante disponíveis no Campus do Arenito da Universidade Estadual de Maringá.

\section{MATERIAL E MÉTODOS}

Para a caracterização hidráulica dos emissores (gotejadores), foi construída uma bancada de ensaios no Laboratório de Hidráulica e Irrigação da Universidade Estadual de Maringá, em Cidade Gaúcha, PR, constituída por: i) bomba centrífuga; ii) tubulação de PVC de $50 \mathrm{~mm}$ de diâmetro; iii) tubulação de PVC de 3/4" de diâmetro; iv) tomadas de pressão e; v) tubos gotejadores. Os tubos gotejadores, com quatro metros de comprimento cada, foram conectados na tubulação de PVC de $3 / 4$ " nas duas extremidades para reduzir o efeito da perda de 
carga ao longo da linha de gotejadores (Figura 1a).

Os ensaios foram realizados com duas amostras obtidas aleatoriamente de tubos gotejadores de regime turbulento e tubos gotejadores de regime autocompensante, totalizando 32 gotejadores ensaiados. Cada amostra, com 16 gotejadores, foi composta de quatro segmentos de tubos gotejadores com quatro metros de comprimento (Figura 1a) e contendo gotejadores espaçados em um metro.

$\mathrm{Na}$ avaliação, os trinta e dois emissores de cada tipo de regime de escoamento de água foram submetidos as pressões de 5, 10, 15, 20, 25 e 30 mca. As pressões de operação foram reguladas com auxílio de registro de gaveta e monitoradas através de manômetro metálico acoplado no início das linhas de tubos gotejadores avaliados (Figura 1b).

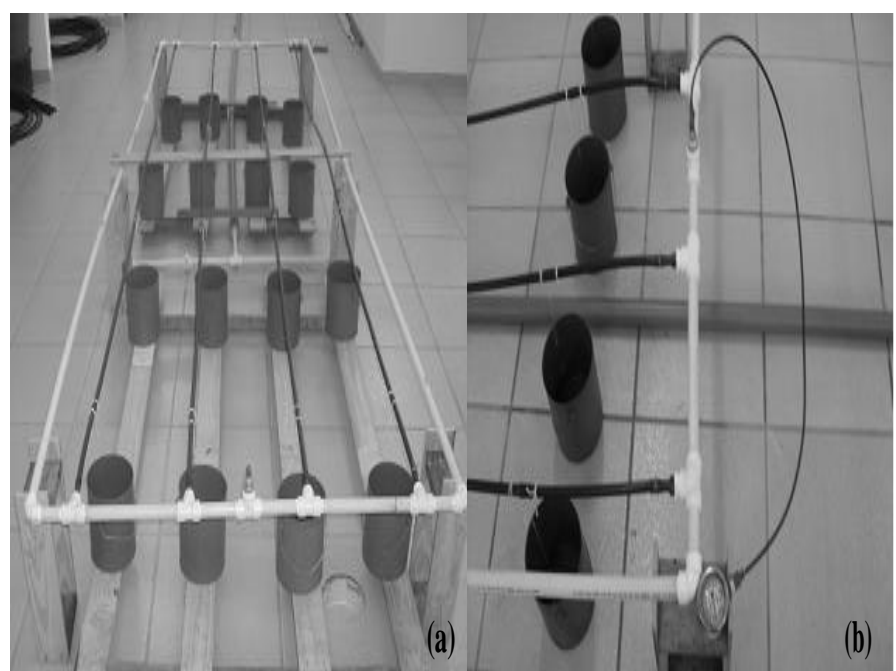

Figura 1. Bancada de ensaios dos tubos gotejadores (a) e tomada de pressão alocada no início das linhas de gotejamento (b).

Para cada amostra de 16 gotejadores, fixada a pressão de operação, simultaneamente, foram coletados os volumes de água de cada gotejador por um tempo de três minutos. A vazão de emissão de cada gotejador, para uma pressão de operação, foi a vazão média dos 32 emissores avaliados. Com os valores de vazão média versus pressão de operação do emissor foi ajustada uma função potencial, dada por:

$$
\mathrm{q}=\mathrm{k} \cdot \mathrm{h}^{\mathrm{x}}
$$

em que q é vazão do emissor $\left(\mathrm{L} \mathrm{h}^{-1}\right)$; $\mathrm{h}$ a pressão de serviço do emissor (mca); k o coeficiente específico de cada emissor e; $\mathrm{x}$ o expoente que caracteriza o escoamento do emissor.

Os valores de vazão dos 32 emissores, obtidos nos ensaios de laboratório para determinar a equação característica dos tubos gotejadores, foram empregados para estabelecer o coeficiente de variação de fabricação (CVF) dos emissores (ASAE, 2003).

No cálculo do comprimento máximo de linhas laterais de irrigação, uma variação de vazão ao longo da linha deve ser considerada para determinar a perda de carga admissível nessa tubulação. Conforme Bernardo et al. (2006), a perda de carga de uma tubulação com múltiplas saídas, corrigida pelo fator $\mathrm{F}$ de múltiplas saídas de Christiansen, pode ser calculada pela equação de Darcy-Weisbach, dada por:

$$
\mathrm{hf}=6,37553 \cdot \mathrm{f} \cdot \frac{\mathrm{Q}^{2}}{\mathrm{D}^{5}} \cdot \mathrm{L} \cdot \mathrm{F}
$$

em que hf é perda de carga na tubulação (mca); f o coeficiente de atrito (decimal); $Q$ a vazão de entrada na linha lateral $\left(\mathrm{L} \mathrm{h}^{-1}\right)$; D o diâmetro interno do tubo (mm); L o comprimento da 
linha lateral (m) e; F o fator de múltiplas saídas de Christiansen (decimal).

No caso de linhas laterais de sistemas de irrigação por gotejamento, o número de saídas (gotejadores) é elevado. Desta forma, o segundo e terceiro termo da equação do fator de múltiplas saídas de Christiansen (F) tendem a zero, podendo a equação ser aproximada por:

$$
\mathrm{F}=\frac{1}{\mathrm{~m}+1}
$$

em que m é o expoente de velocidade ou vazão da expressão de perda de carga.

$O$ coeficiente de atrito (f) da equação de Darcy-Weisbach, para tubos operando em regime de escoamento turbulento liso, pode ser calculado pela equação de Blasius (MANTOVANI et al., 2009) e para a viscosidade cinemática da água de $10^{-6} \mathrm{~m} \mathrm{~s}^{-2}$ (temperatura $20^{\circ} \mathrm{C}$ ), a equação do comprimento máximo da linha lateral é estimada por:

$$
\begin{gathered}
\mathrm{L}_{\text {max }}=1,9135 \cdot \mathrm{D}^{1,7273} \cdot \mathrm{hf}_{\mathrm{adm}} 0,3636 \cdot\left(\frac{\mathrm{e}}{\mathrm{q}}\right)^{0,6364} \\
\text { sendo: } \mathrm{q}=\frac{\mathrm{Q}}{\mathrm{N}}=\frac{\mathrm{Q} \cdot \mathrm{e}}{\mathrm{L}}
\end{gathered}
$$

em que $\mathrm{L}_{\mathrm{max}}$ é o comprimento máximo da linhas lateral (m); $\mathrm{hf}_{\mathrm{adm}}$ a perda de carga admissível na linha lateral (mca); e o espaçamento entre emissores (m); $\mathrm{N}$ o número de emissores na linha lateral e; q a vazão de um emissor $\left(\mathrm{L} \mathrm{h}^{-1}\right)$.

A perda de carga admissível, apresentada na expressão 5, pode ser calculada pela variação máxima da vazão na linha lateral. Expressando a variação da vazão em termos da variação de pressão entre o início e o final de uma linha lateral em nível, tem-se a equação:

$$
\mathrm{hf}_{\mathrm{adm}}=\mathrm{h}_{\text {var }} \cdot \mathrm{h}=\left(1-\frac{\mathrm{h}_{\text {mín }}}{\mathrm{h}_{\text {máx }}}\right) \cdot \mathrm{h}
$$

em que $h_{\text {var }}$ é a variação de pressão na linha

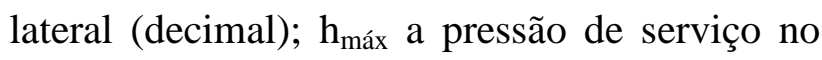

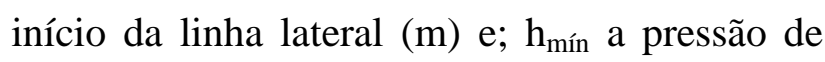
serviço no final da linha lateral $(\mathrm{m})$.

A relação $\left(\mathrm{h}_{\text {mín }} / \mathrm{h}_{\text {máx }}\right)$, dada na equação 6 , pode ser obtida a partir da equação característica da vazão do emissor por:

$$
\frac{\mathrm{q}_{\text {máx }}}{\mathrm{q}_{\text {mín }}}=\frac{\mathrm{k} \cdot \mathrm{h}_{\text {máx }}^{\mathrm{n}}}{\mathrm{k} \cdot \mathrm{h}_{\text {mín }}^{\mathrm{n}}}=\left(\frac{\mathrm{h}_{\text {máx }}}{\mathrm{h}_{\text {mín }}}\right)^{\mathrm{n}}
$$

em que q qáx é a vazão do primeiro emissor $\left(\mathrm{L} \mathrm{h}^{-1}\right)$ e; $\mathrm{q}_{\text {mín }}$ a vazão do último emissor $\left(\mathrm{L} \mathrm{h}^{-1}\right)$.

No cálculo do comprimento máximo da linha lateral trabalhando em nível, para os dois tipos de tubos gotejadores, foram consideradas pressões de operação de 5,10 e 20 mca e variações de vazão de 5, 10 e $15 \%$.

A avaliação no campo dos tubos gotejadores de regime turbulento e autocompensante foi realizada em uma área experimental da Universidade Estadual de Maringá, em Cidade Gaúcha, PR. Esta área conta com um cabeçal de controle, para filtragem da água e regulagem da pressão e vazão do sistema, uma linha de derivação com seis saídas, instalada no sentido da declividade de $3 \%$ e espaçadas em $6 \mathrm{~m}$, para acoplamento de linhas laterais de irrigação. 
Os ensaios de distribuição de água, para três pressões de operação $(5,10$ e 15 mca), foram realizados com seis linhas laterais de 61 m de comprimento instaladas em nível e com emissores espaçados em um metro (Figura 2a). As avaliações foram realizadas em períodos distintos, primeiramente com os tubos gotejadores de regime turbulento e posteriormente com os tubos gotejadores autocompensante.

Após a estabilização da pressão de serviço, medida com manômetro metálico na entrada da segunda linha lateral, considerando o sentido da declividade, foi medida a pressão de operação no final da segunda linha lateral e foram determinadas as vazões aplicadas das seis linhas laterais. Em cada linha lateral, foram coletados, por um tempo de dois minutos os volumes de água (Figura 2b) em quatro pontos de emissão, determinados pelo número de emissores na linha lateral por: primeiro; $1 / 3 ; 2 / 3 \mathrm{e}$ último emissor.
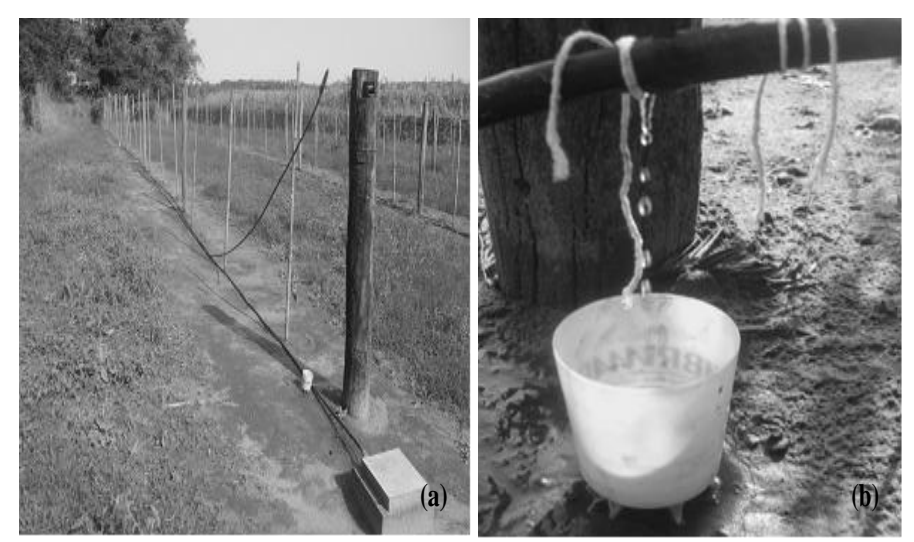

Figura 2. Disposição dos tubos gotejadores no campo (a) e coleta de água em um ponto de amostragem (b).

Os valores de vazão coletados nos pontos de amostragem, em função da pressão de operação, foram empregados para determinar a uniformidade de distribuição da água (UD) do sistema de irrigação através da equação:

$$
\mathrm{UD}=\frac{\overline{\mathrm{q}}_{25}}{\overline{\mathrm{q}}} \cdot 100
$$

em que UD é a uniformidade de distribuição de água $(\%)$ e; $\overline{\mathrm{q}}_{25}$ a vazão média dos $25 \%$ menores valores de vazão $\left(\mathrm{L} \mathrm{h}^{-1}\right)$.

$\mathrm{Na}$ determinação do grau de adequação e das eficiências de irrigação é necessário definir o volume de água requerido pela cultura. Para efeito de cálculo, foi considerado que o volume requerido pela cultura é de $15 \mathrm{~L}$. Desta forma, a partir da relação entre as vazões coletadas em cada ponto de amostragem pela vazão média coletada, foi determinado o volume de água aplicado por cada emissor:

$$
\mathrm{Va}_{\mathrm{i}}=\mathrm{Vr} \cdot \frac{\mathrm{q}_{\mathrm{i}}}{\overline{\mathrm{q}}}
$$

em que $\mathrm{Va}_{\mathrm{i}}$ é o volume de água aplicado por um emissor de índice i (L); Vr o volume requerido pela cultura (L) e; $q_{i}$ a vazão aplicada por um emissor de índice $\mathrm{i}\left(\mathrm{L} \mathrm{h}^{-1}\right)$.

Assumindo que os dados de volumes de água aplicados seguem uma distribuição normal, o procedimento utilizado para o cálculo da fração de área que recebe no mínimo o volume de água requerido, foi feito determinando-se a área subentendida pela curva da distribuição normal. Desta forma, a probabilidade do valor de volume requerido pela cultura ser excedido pode ser calculado por: 


$$
\mathrm{P}\left(\mathrm{Vr} \geq \mathrm{Va}_{\mathrm{i}}\right)=\int_{\mathrm{Vr}_{\mathrm{r}}}^{\infty} \frac{1}{\mathrm{~s} \cdot \sqrt{2 \cdot \pi}} \cdot \mathrm{e}^{-0,5\left[\frac{\left(\mathrm{Va}_{\mathrm{i}}-\overline{\mathrm{V}}\right)}{\mathrm{s}}\right]} \cdot \mathrm{dVa}
$$

em que, $\mathrm{P}\left(\mathrm{Vr} \geq \mathrm{Va}_{\mathrm{i}}\right)$ é a probabilidade do volume de água requerido ser maior que o volume de água aplicado; $\overline{\mathrm{V}}$ o volume médio aplicado (L); s o desvio padrão (L).

A equação 10 pode ser resolvida a partir da distribuição normal padrão, que utilizada a chamada variável reduzida ou padrão " $z$ " ou a partir de aplicativos computacionais. Com este intuito, foi utilizado a função Dist.Norm da planilha eletrônica Excel, que requer os dados de volume aplicado, volume médio aplicado e desvio padrão dos volumes aplicados para retornar ao valor da probabilidade do volume de água requerido ser excedido.

Com os valores de volumes de água aplicados, conforme Soccol et al. (2002) e Frizzone e Dourado Neto (2003), foram calculadas as eficiências de aplicação e armazenamento de água no solo e as perdas por percolação de água para valores de volume requerido entre 1 a $40 \mathrm{~L}$, com intervalos de $1 \mathrm{~L}$, para os seis ensaios de distribuição de água.

\section{RESULTADOS E DISCUSSÃO}

Os valores de vazão e coeficiente de variação de fabricação (CVF), em função da pressão de operação para os tubos gotejadores de regime turbulento, são apresentados na Figura 3. Pelo alto coeficiente de determinação $\left(\mathrm{R}^{2}=0,9987\right)$, apresentado na Figura 3a, é evidenciado o comportamento potencial dos valores da vazão em relação à pressão de serviço compreendida entre 5 a 30 mca. Nota-se que o expoente da equação característica da vazão tende a 0,5 , caracterizando o gotejador como um emissor de regime turbulento (KELLER; BLIESNER, 1990; CABELLO, 1996; FRIZZONE et al., 1998).

$\mathrm{Na}$ Figura $3 \mathrm{~b}$ observa-se que os valores de CVF dos gotejadores de regime turbulento, para as diferentes pressões de operação, foram todos inferiores a 5\%, com média de 2,53\%. Conforme a ASAE (2003), emissores com CVF menores a $5 \%$ são classificados como excelentes e demonstram alta qualidade no processo de fabricação.

O comportamento da vazão, em relação a faixa de pressão de serviço entre 5 a 30 mca para o tubo gotejador autocompensante é mostrado na Figura 4a. Observa-se nesta figura que, independente da pressão de operação, a vazão manteve-se praticamente constante com um valor médio de $3,98 \mathrm{~L} \mathrm{~h}^{-1}$.

(a)

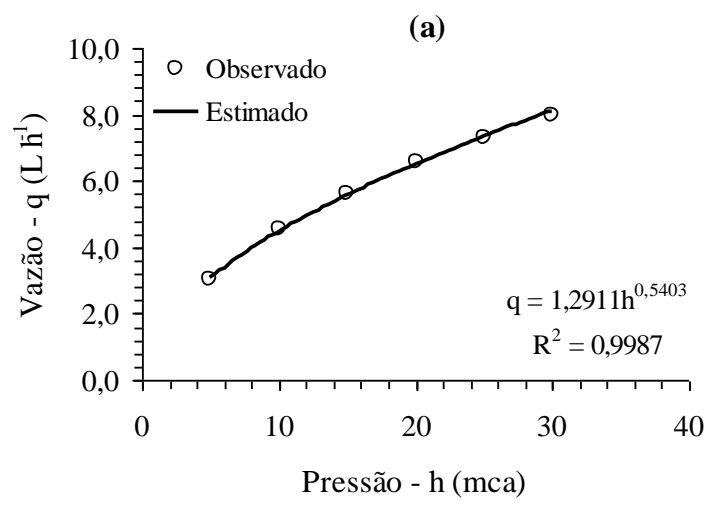




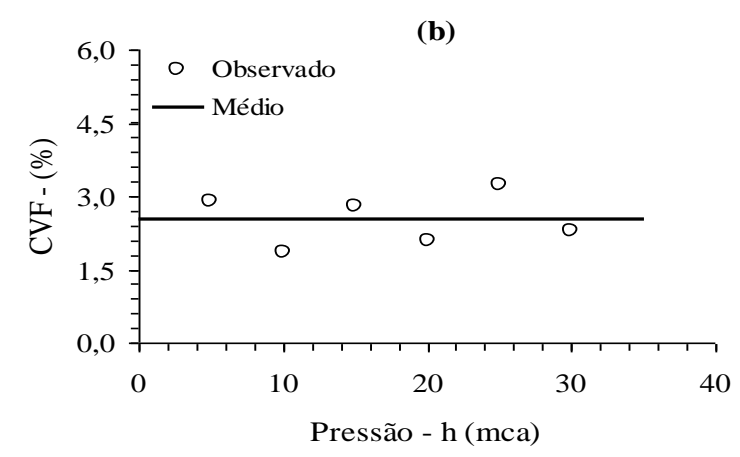

Figura 3. Vazão de emissão (a) e coeficiente de variação de fabricação (b) em função da pressão de serviço dos tubos gotejadores de fluxo turbulento.

Os valores de CVF do tubo gotejador autocompensante, para as diferentes pressões de ensaio, são apresentados na Figura 4b. O valor médio do efeito dos fatores construtivos do emissor foi de 3,98\%, demonstrando assim qualidade do ponto de vista do processo de fabricação do gotejador, que, pela classificação proposta pela ASAE (2003), enquadra-se como excelente.

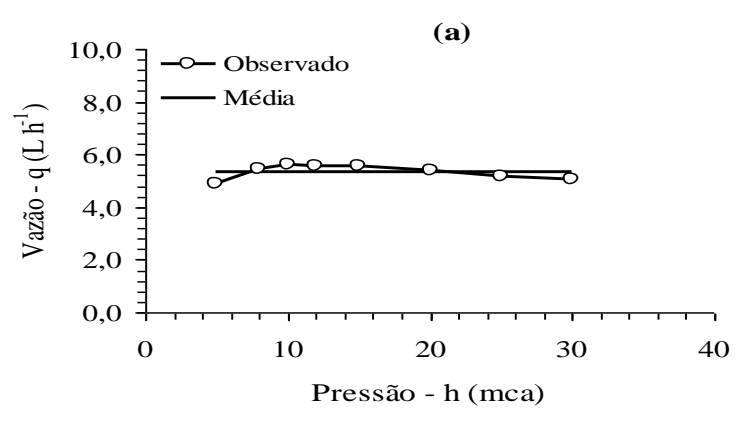

(b)

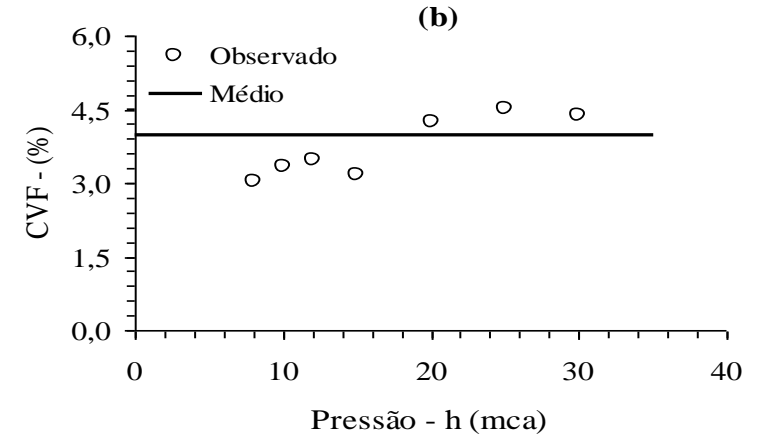

Figura 4. Vazão de emissão (a) e coeficiente de variação de fabricação (b) em função da pressão de serviço dos tubos gotejadores de fluxo turbulento.
Para um melhor ajuste da equação potencial do tubo gotejador autocompensante (Figura 5), os valores de vazão obtidos em laboratório, para a faixa de pressão entre 5 a 15 mca, foram utilizados, obtendo-se um expoente da equação de 0,1116 e um coeficiente de determinação de 75,01\%. Conforme Keller e Bliesner (1990) e Cabello (1996), expoente da equação característica tendendo a zero é característico de emissores autocompensantes.

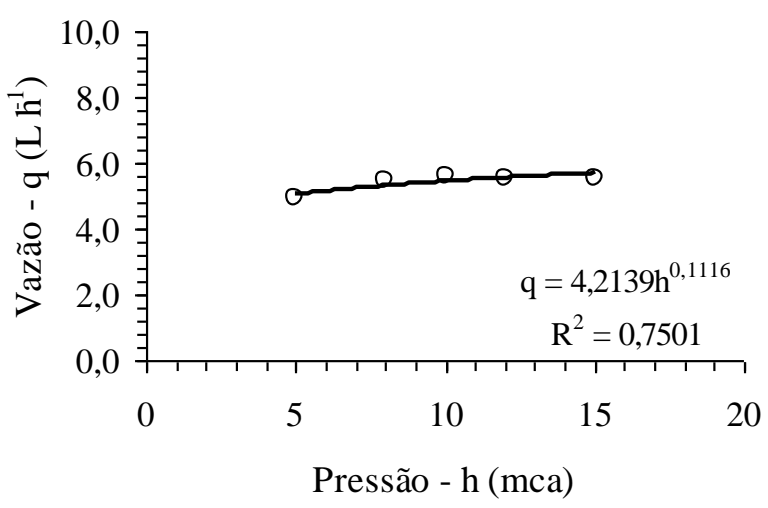

Figura 5. Vazão de emissão em função da pressão de serviço dos tubos gotejadores de fluxo autocompensante.

Na Tabela 1 são apresentados os valores calculados de comprimento máximo da linha lateral dos tubos gotejadores de regime turbulento com diâmetro interno de 13,59 mm e autocompensantes com diâmetro interno de 13,03 mm. Considerando os expoentes da equação de vazão dos emissores de regime turbulento $(\mathrm{x}=0,5403)$ e autocompensante $(\mathrm{x}=$ 0,1116), as variações de pressão para o tubo gotejador de regime autocompensante foram maiores, proporcionando comprimento de linha lateral mais extenso em relação aos tubos gotejadores de regime turbulento. 
De acordo com Cabello (1996) e Bernardo et al. (2006), normalmente, uma variação de vazão de $10 \%$ ao longo da linha lateral de sistemas de irrigação localizada é empregada no seu dimensionamento. Para variação de vazão de $10 \%$ (Tabela 1) obteve-se um comprimento máximo de linha lateral de 80 metros para gotejadores de regime turbulento e de 104 metros para gotejadores de regime autocompensante. O emprego de variações de vazão ao longo da linha lateral superiores ou inferiores a $10 \%$, dependerá, principalmente, do valor econômico da cultura; culturas com baixos retornos econômicos, uma maior variação na vazão pode ser admitida, gerando linhas laterais mais compridas (Tabela 1).

Tabela 1. Valores de comprimento máximo da linha lateral em relação a variação de vazão e variação de pressão para tubos de fluxo turbulento e autocompensante.

\begin{tabular}{|c|c|c|c|c|}
\hline \multirow[b]{2}{*}{$\begin{array}{c}\text { Variação } \\
\text { na vazão } \\
- \\
\Delta q(\%)\end{array}$} & \multicolumn{2}{|c|}{$\begin{array}{l}\text { Gotejador } \\
\text { turbulento }\end{array}$} & \multicolumn{2}{|c|}{$\begin{array}{c}\text { Gotejador } \\
\text { autocompensante }\end{array}$} \\
\hline & $\begin{array}{c}\text { Variaçã } \\
\text { o na } \\
\text { pressão } \\
- \\
\Delta \mathrm{h}(\%)\end{array}$ & $\begin{array}{c}\text { Compri } \\
\text { mento } \\
\text { máximo } \\
-\mathrm{L}_{\max } \\
(\mathrm{m}) \\
\end{array}$ & $\begin{array}{c}\text { Variação } \\
\text { na } \\
\text { pressão - } \\
\Delta \text { h (\%) }\end{array}$ & $\begin{array}{c}\text { Compri } \\
\text { mento } \\
\text { máximo } \\
- \\
\mathrm{L}_{\max }(\mathrm{m}) \\
\end{array}$ \\
\hline 5 & 8,6 & 63 & 35,4 & 87 \\
\hline 10 & 16,2 & 80 & 57,4 & 104 \\
\hline 15 & 22,8 & 90 & 71,4 & 112 \\
\hline 20 & 28,6 & 98 & 80,5 & 117 \\
\hline
\end{tabular}

As variações de pressão e vazão observadas no campo com as linhas laterais de 61 metros de comprimento, para os tubos gotejadores de regime turbulento e autocompensantes, respectivamente, são apresentadas nas Tabelas 2 e 3. Para uma pressão de $10 \mathrm{mca}$ na entrada da linha lateral e operação dos gotejadores (MANTOVANI et al., 2009), observou-se uma variação de vazão igual a $4,9 \%$ para os tubos gotejadores de regime turbulento e de $2,3 \%$ para os tubos gotejadores de regime autocompensante.

Tabela 2. Variação de pressão e vazão na segunda linha lateral observadas no campo para tubos gotejadores de fluxo turbulento.

\begin{tabular}{cccc}
$\begin{array}{c}\text { Pressão } \\
\text { média: } \\
\text { entrada } \\
\begin{array}{c}\text { linha }-\mathrm{h}_{\text {máx }} \\
(\mathrm{mca})\end{array}\end{array}$ & $\begin{array}{c}\text { Pressão } \\
\text { média: } \\
\text { final da } \\
\text { linha }- \\
\mathrm{h}_{\text {mín }}(\mathrm{mca})\end{array}$ & $\begin{array}{c}\text { Variação na } \\
\text { pressão }-\Delta \mathrm{h} \\
(\%)\end{array}$ & $\begin{array}{c}\text { Variação na } \\
\text { vazão }-\Delta \mathrm{q} \\
(\%)\end{array}$ \\
\hline 5 & 4,07 & 18,7 & 11,8 \\
10 & 9,15 & 8,5 & 4,9 \\
15 & 14,07 & 6,2 & 3,5 \\
\hline
\end{tabular}

Pode-se inferir através das Tabelas 2 e 3 que devido ao expoente $\mathrm{x}$ da equação característica da vazão dos emissores estudados, as menores variações de vazão encontradas foram para os tubos gotejadores de regime autocompensante. Conforme Cabello (1996), os gotejadores de regime autocompensante apresentam menor sensibilidade as variações de pressão ao longo da linha lateral.

Tabela 3. Variação de pressão e vazão na segunda linha lateral observadas no campo para tubos gotejadores de fluxo autocompensante.

\begin{tabular}{cccc}
\hline $\begin{array}{c}\text { Pressão } \\
\text { média: } \\
\text { entrada } \\
\begin{array}{c}\text { linha }-\mathrm{h}_{\text {máx }} \\
(\text { mca })\end{array}\end{array}$ & $\begin{array}{c}\text { Pressão } \\
\text { média: } \\
\text { final da } \\
\text { linha }- \\
\mathrm{h}_{\text {mín }}(\text { mca })\end{array}$ & $\begin{array}{c}\text { Variação na } \\
\text { pressão }-\Delta \mathrm{h} \\
(\%)\end{array}$ & $\begin{array}{c}\text { Variação na } \\
\text { vazão }-\Delta \mathrm{q} \\
(\%)\end{array}$ \\
\hline 5 & 3,20 & 36,0 & \\
10 & 8,13 & 18,7 & 2,3 \\
15 & 13,63 & 9,1 & 1,1 \\
\hline
\end{tabular}


Para a pressão de entrada de 10 mca na linha lateral de $61 \mathrm{~m}$ de comprimento do tubo gotejador de regime turbulento, observou-se uma variação de vazão de 4,9\% (Tabela 2), valor muito próximo a 5\% empregado para estimar o comprimento máximo de $63 \mathrm{~m}$ da linha lateral de tubos lisos (Tabela 1). Esta pequena diferença reflete um baixo valor de perda de carga localizada devido a inserção do emissor no tubo.

$\mathrm{Na}$ linha lateral de $61 \mathrm{~m}$ de comprimento do tubo gotejador autocompensante, uma variação de $5,1 \%$ foi observada para a pressão de entrada de 5 mca (Tabela 2). Para a variação de vazão de 5\% (Tabela 1) deste tubo gotejador, estimou-se um comprimento máximo de $87 \mathrm{~m}$ de linha lateral para um tubo sem perdas de cargas localizadas (Tabela 1). Desta forma, para esse tipo de emissor as de perdas de cargas localizadas foram mais expressivas.

A uniformidade de distribuição de água para os dois tipos de tubos gotejadores estudados é apresentada na Tabela 4. Os valores obtidos nos ensaios estão dentro ou acima da faixa de uniformidade (85 a 90\%) recomendada pela ASAE (2003). Conforme Cabello (1996), altos valores de uniformidade de distribuição de água são obtidos com emissores com baixos valores de CVF e corretos dimensionamentos hidráulicos das linhas de irrigação.
Tabela 4. Uniformidade de distribuição de água do sistema de irrigação por gotejamento operando com gotejadores de fluxo turbulento e autocompensante.

\begin{tabular}{ccc}
\hline Pressão - h (mca) & turbulento & autocompensante \\
\hline 5 & 90,8 & 84,9 \\
10 & 95,9 & 93,5 \\
15 & 96,0 & 93,1 \\
\hline
\end{tabular}

$\mathrm{Na}$ Tabela 4, observa-se que a maior variação de vazão (Tabela 2 e 3), para a pressão de entrada de 5 mca nas linhas laterais dos tubos gotejadores de regime turbulento e autocompensante, proporcionaram os menores valores de uniformidade de distribuição de água. Para as pressões de entrada nas linhas laterais de 10 e 15 mca, praticamente, não houve diferença entre os valores de uniformidade de distribuição de água nos dois tipos de tubos gotejadores estudados. Silva et al. (2012) observaram que pressões de operação maiores que $15 \mathrm{mca}$ promoveram decréscimos na uniformidade de aplicação de água do sistema de irrigação. Estes resultados ratificam o emprego da pressão de 10 mca recomendada por Cabello (1996), Bernardo et al. (2006) e Mantovani et al. (2009) para operação de linhas laterais de sistemas de irrigação por gotejamento.

O grau de adequação do sistema de irrigação está relacionado com a uniformidade de distribuição de água, quanto melhor for a uniformidade, menor serão os volumes de água em excesso e déficit na área irrigada (CLEMMENTS; SOLOMON, 1997). Este comportamento é evidenciado na Figura 6, que para a menor uniformidade de distribuição de 


\section{LOCALIZADA}

água do sistema de irrigação (UD $=84,9 \%$ ), operando com o tubo gotejador autocompensante na pressão de $5 \mathrm{mca}$, verificou-se uma maior variação nos volumes de água aplicados. Já para as pressões de 10 e 15 mca, para os dois tipos de emissores, as curvas são mais planas, acarretando em menores perdas de água por percolação profunda.

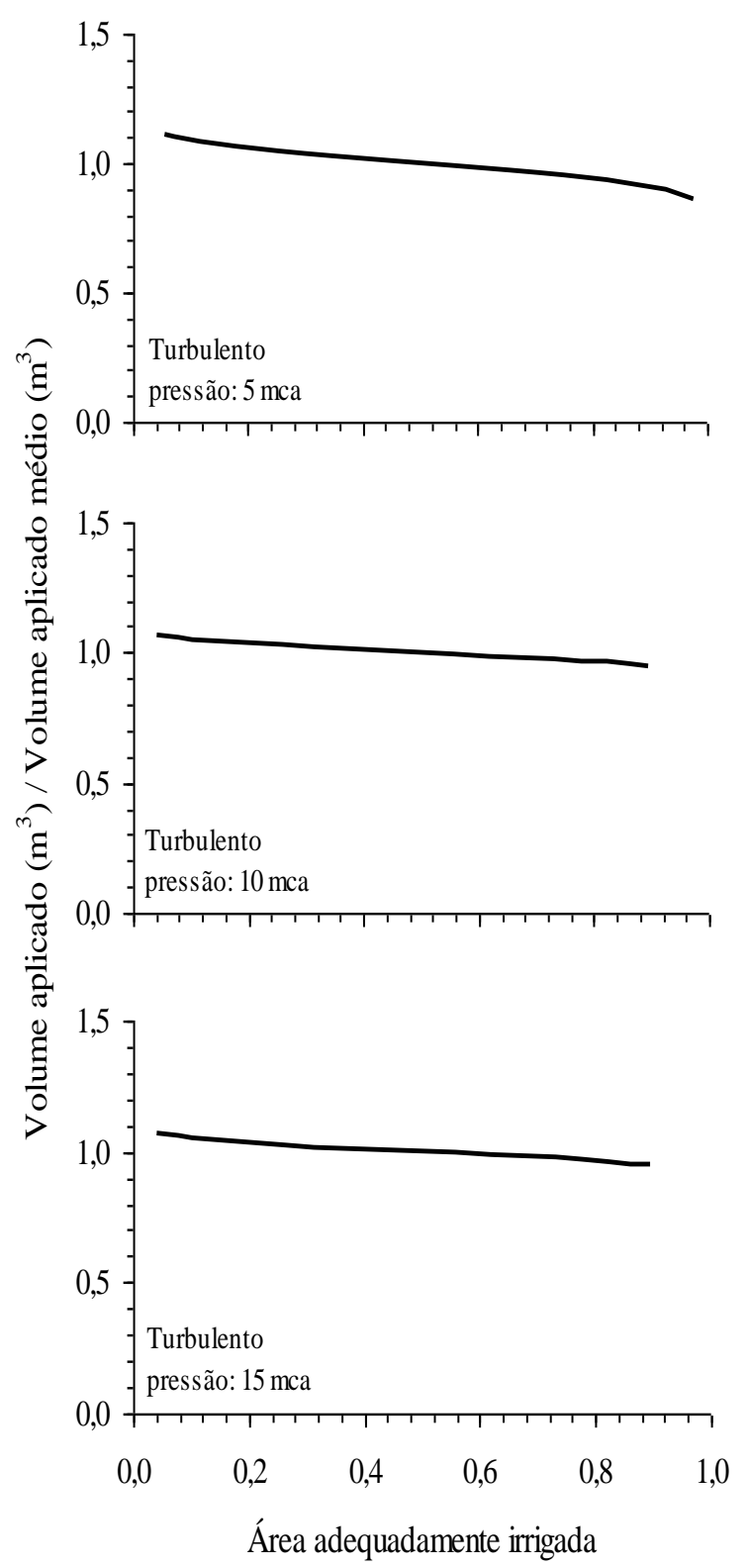



Figura 6. Área adequadamente irrigada do sistema de irrigação por gotejamento operando com gotejadores de fluxo turbulento e autocompensante.

$\mathrm{Na}$ Tabela 5 são apresentadas as eficiências de aplicação, as eficiências de armazenamento e as perdas por percolação. Os altos valores de eficiência de aplicação, para os dois tipos de tubos gotejadores, indicam que praticamente todo o volume de água aplicado foi armazenado na zona radicular de uma 
cultura hipotética, resultando em baixos valores de perdas de água por percolação profunda. Para a eficiência de armazenamento, nos dois tipos de tubos gotejadores avaliados, foram verificados valores superiores a 94\%, ou seja, o volume de água aplicado atende praticamente o volume total de água requerido.

Tabela 5. Eficiências de aplicação (Ea), armazenamento (Es) e perdas por percolação (Pp), para um volume requerido de $15 \mathrm{~L}$, do sistema de irrigação por gotejamento operando com gotejadores de regime turbulento $\mathrm{e}$ autocompensante.

\begin{tabular}{|c|c|c|c|c|c|c|}
\hline \multirow{2}{*}{$\begin{array}{c}\text { Pressão } \\
-\mathrm{h} \\
(\mathrm{mca})\end{array}$} & \multicolumn{3}{|c|}{ Turbulento } & \multicolumn{3}{|c|}{ Autocompensante } \\
\hline & $\mathrm{Ea}$ & Es & $\mathrm{Pp}$ & $\mathrm{Ea}$ & Es & $\mathrm{Pp}$ \\
\hline 5 & 0,971 & 0,971 & 0,028 & 0,940 & 0,940 & 0,060 \\
\hline 10 & 0,982 & 0,982 & 0,018 & 0,978 & 0,978 & 0,022 \\
\hline 15 & 0,985 & 0,985 & 0,015 & 0,976 & 0,976 & 0,024 \\
\hline
\end{tabular}

O comportamento das eficiências de aplicação, de armazenamento e as perdas por percolação em função da relação entre volume requerido pelo volume aplicado, conforme descrito pela American Society of Civil Engineers (1978), são apresentados na Figura 7. Para as três pressões de operação aplicadas nos dois tipos de tubos emissores é observado que quando a eficiência de armazenamento é máxima $($ Es $=100 \%)$, ou seja, todos os pontos da área recebem o volume de água requerido ou mais, ocorrerão perdas por percolação que leva a um baixo valor de eficiência de aplicação de água. A medida que o volume de água requerido aumenta, menor serão as perdas de água por percolação e maior será o valor da eficiência de aplicação de água (Figura 7).
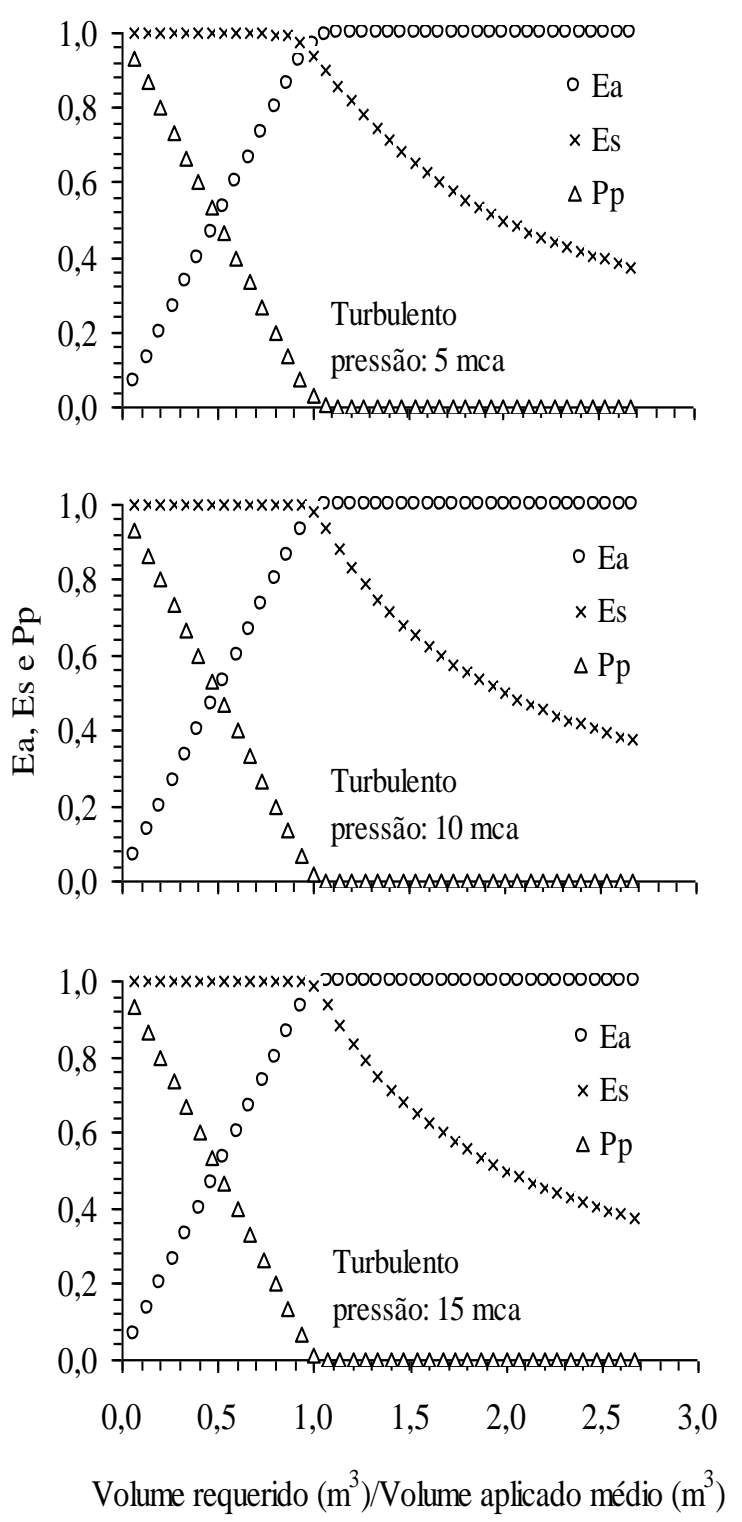

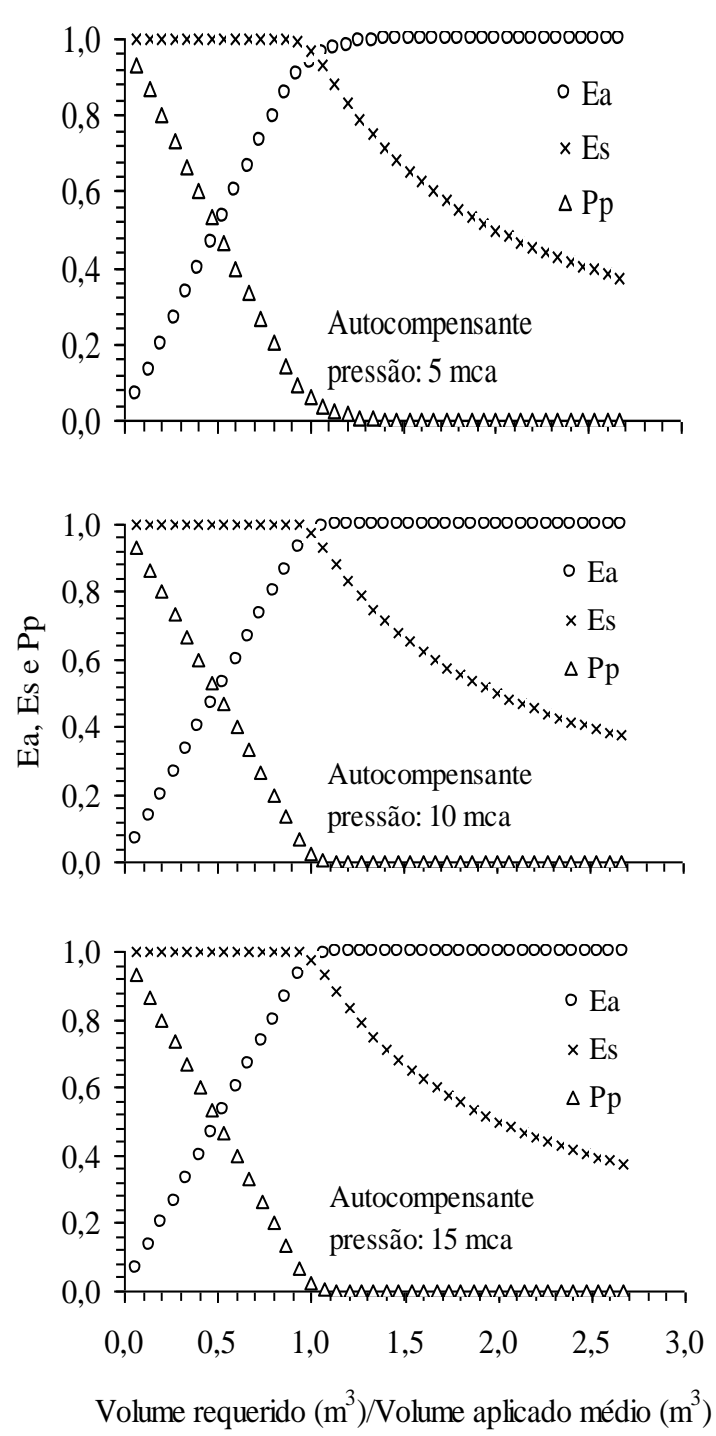

Figura 7. Eficiência de aplicação (Ea), armazenamento (Es) e perdas por percolação $(\mathrm{Pp})$ do sistema de irrigação por gotejamento operando com gotejadores de fluxo turbulento e autocompensante.

\section{CONCLUSÕES}

Com base nos resultados obtidos concluise que: i) os expoentes das equações características atenderam o regime de escoamento dos dois tipos de tubos emissores estudados. ii) os tubos gotejadores avaliados apresentam excelente qualidade de fabricação (CVF < 5\%); iii) pressão de operação nas linhas laterais superior a 10 mca não promoveu uma melhora na distribuição de água para os dois tipos de tubos gotejadores.

\section{REFERÊNCIAS BIBLIOGRÁFICAS}

ASAE. AMERICAN SOCIETY OF AGRICULTURAL ENGINEERS. Design and installation of microirrigation systems. St. Joseph: ASAE Standards engineering practices data: EP 405.1, 2003.

ASCE. AMERICAN SOCIETY OF CIVIL ENGINEERS. Describing irrigation efficiency and uniformity. Journal of Irrigation and Drainage Engineering, New York, v.104, n. 1, p. 35-41, 1978.

BERNARDO, S.; SOARES, A.A.; MANTOVANI, E.C. Manual de irrigação. Viçosa, MG: UFV, 2006.

BORSSOI, A. L.; VILAS BOAS, M. A.; REISDÖRFER, M; HERNÁNDEZ, R. H.; FOLLADOR, F. C. Water application uniformity and fertigation in a dripping irrigation set. Engenharia Agrícola, Jaboticabal, v. 32, n. 4, p. 718-726, 2012.

CABELLO, F. P. Riegos localizados de alta frecuencia: goteo, microaspersión, exudación. 3.ed. Madrid: Ediciones Mundi-Prensa, 1996. $513 \mathrm{p}$.

CLEMMENS, A. J.; SOLOMON, K. H. Estimation of global irrigation distribution uniformity. Journal of Irrigation and 
Drainage Engineering, New York, v. 123, n. 6, p.454-461, 1997.

FRIZZONE, J. A.; VIEIRA, A. T.; PAZ, V. P. S.; BOTREL, T. A. Caracterização hidráulica de um tubo gotejador. Revista Brasileira de Engenharia Agrícola e Ambiental, Campina Grande, v. 2, n. 3, p. 278-283, 1998.

FRIZZONE, J. A.; DOURADO NETO, D. Avaliação de sistemas de irrigação. In: MIRANDA, J. H.; PIRES, R. C. M. Irrigação. Jaboticabal: Sociedade Brasileira de Engenharia Agrícola, 2003. p. 573-651.

HOLZAFEL, E. A.; PARDO, X. M.; PAZ, V. P. S.; RODRIGUEZ, A.; ORREGO, X. C.; LOPEZ, M. A. Análisis técnico-econômico para selección de aspersores. Revista Brasileira de Engenharia Agrícola e Ambiental, Campina Grande, v. 11, n. 6, p. 557-563, 2007.

KELLER, J., BLIESNER, R. D. Sprinkle and Trickle Irrigation. Van Nostrand Reinhold, New York, 1990. 652 p.

LAPERUTA NETO, J; CRUZ, R. L.; LAPERUTA FILHO, J; PLETSCH, T. A. Perda de carga em mangueiras gotejadoras novas e usadas. Irriga, Botucatu, v. 16, n. 3, p. 329-338, 2011.

MANTOVANI, E.C.; BERNARDO, S.; PALARETTI, L. F. Irrigação: Princípios e métodos. 3.ed. Viçosa: UFV, 2009. 355 p.

PIRES, R. C. M.; ARRUDA, F. B.; SAKAI, E.; CALHEIROS, R. O.; BRUNINI, O. Agricultura irrigada. Revista tecnologia $e$ inovação agropecuária, São Paulo, v. 1, n. 1, p. 98-111, 2008.

SARAIVA, K. R.; SOUZA, F. Estatística sobre irrigação nas regiões sul e sudeste do Brasil segundo o censo agropecuário 2005-2006. Irriga, Botucatu, v. 17, n. 2, p. 168-176, 2012.

SILVA, L. P.; SILVA, M. M.; CORREA, M. M.; SOUZA, F. C. D.; SILVA, E. F. F. Desempenho de gotejadores autocompensantes com diferentes efluentes de esgoto doméstico. Revista Brasileira de Engenharia Agrícola e Ambiental, Campina Grande, v. 16, n. 5, p. 480-486, 2012.

SOCCOL, O. J.; ULLMANN, M. N.; FRIZZONE, J. A. Performance analysis of a trickle subunit installed in an apple orchard. Brazilian archives of biology and technology, Curitiba, v. 45, n. 4, p. 525-530, 2002.

SOUZA, W. J.; BOTREL, T. A.; COELHO, R. D.; NOVA, N. A. V. Irrigação localizada subsuperficial: gotejador convencional e novo protótipo. Revista Brasileira de Engenharia Agrícola e Ambiental, Campina Grande, v. 16, n. 8, p. 811-819, 2012. 